\title{
Preoperative levels of natriuretic peptides and the incidence of postoperative atrial fibrillation after noncardiac surgery: a prospective cohort
} study

\author{
Wojciech Szczeklik PhD, Yannick LeManach PhD, ${ }^{*}$ Jakub Fronczek MD, Kamil Polok MD, David Conen MD, \\ Finlay A. McAlister PhD, Sadeesh Srinathan PhD, Pablo Alonso-Coello PhD, Bruce Biccard PhD, \\ Emmanuelle Duceppe MD, Diane Heels-Ansdell MSc, Jacek Górka MD, Shirley Pettit MSc, Pavel S. Roshanov MD, \\ P.J. Devereaux PhD
}

Cite as: CMAJ 2020 December 7;192:E1715-22. doi: 10.1503/cmaj.200840

\begin{abstract}
BACKGROUND: Postoperative atrial fibrillation (POAF) is associated with clinically significant short- and long-term complications after noncardiac surgery. Our aim was to describe the incidence of clinically important POAF after noncardiac surgery and establish the prognostic value of $\mathrm{N}$-terminal pro-brain-type natriuretic peptide (NT-proBNP) in this context.
\end{abstract}

METHODS: The Vascular events In noncardiac Surgery patlents cOhort evaluatioN (VISION) Study was a prospective cohort study involving patients aged 45 years and older who had inpatient noncardiac surgery that was performed between August 2007 and November 2013. We determined 30-day incidence of clinically important POAF (i.e., resulting in angina, congestive heart failure, symptomatic hypotension or requiring treatment) using logistic regression models to analyze the association between preoperative NT-proBNP and POAF.

RESULTS: In 37664 patients with no history of atrial fibrillation, we found that the incidence of POAF was $1.0 \%$ (95\% confidence interval $[\mathrm{Cl}] 0.9 \%-$ $1.1 \%$; 369 events); $3.2 \%$ (95\% Cl 2.3\%$4.4 \%)$ in patients undergoing major thoracic surgery, $1.3 \%(95 \% \mathrm{Cl} 1.2 \%-1.5 \%)$ in patients undergoing major nonthoracic surgery and $0.2 \%(95 \% \mathrm{Cl} 0.1 \%-$ $0.3 \%$ ) in patients undergoing low-risk surgery. In a subgroup of 9789 patients with preoperative NT-proBNP measurements, the biomarker improved the pre- diction of POAF risk over conventional prognostic factors (likelihood ratio test $p<0.001$; fraction of new information from NT-proBNP was 16\%). Compared with a reference NT-proBNP measurement set at $100 \mathrm{ng} / \mathrm{L}$, adjusted odds ratios for the occurrence of POAF were $1.31(95 \% \mathrm{Cl} 1.15-1.49)$ at $200 \mathrm{ng} / \mathrm{L}, 2.07$ (95\% Cl 1.27-3.36) at $1500 \mathrm{ng} / \mathrm{L}$ and 2.39 (95\% Cl 1.26-4.51) at $3000 \mathrm{ng} / \mathrm{L}$.

INTERPRETATION: We determined that the incidence of clinically important POAF after noncardiac surgery was $1.0 \%$. We also found that preoperative NT-proBNP levels were associated with POAF independent of established prognostic factors. Trial registration: ClinicalTrials.gov, no. NCT00512109 ostoperative atrial fibrillation (POAF) is the most common arrhythmia that occurs after noncardiac surgery $^{1}$ and is associated with prolonged hospital stays and an increased risk of stroke and death, ${ }^{2-8}$ both of which raise societal costs. ${ }^{9}$ The reported incidence of POAF ranges from $3.7 \%$ to $21.0 \%$ in patients undergoing thoracic surgery $^{3,10}$ and from less than $0.5 \%$ to $10.0 \%$ in patients undergoing noncardiac, nonthoracic surgery (Appendix 1, Supplementary Table 1, available at www.cmaj.ca/lookup/doi/10.1503/ cmaj.200840/tab-related-content). ${ }^{6,11}$
Brain-type natriuretic peptide (BNP) is a neurohormone released by cardiomyocytes in response to volume and pressure overload. ${ }^{12}$ This biomarker has an established role in the diagnosis and management of heart failure and in predicting cardiovascular morbidity and mortality. ${ }^{13}$ Although natriuretic peptides have been shown to predict new-onset atrial fibrillation (AF) in the nonsurgical setting reliably, ${ }^{14-18}$ their value in predicting POAF after noncardiac surgery is unclear. ${ }^{19-21}$

The Vascular events In non-cardiac Surgery patlents cOhort evaluatioN (VISION) Study was a prospective international cohort 
study involving a representative sample of adults who underwent noncardiac surgery that required an overnight hospital stay. One of the predefined objectives in VISION was to determine the incidence of new-onset, clinically important POAF. We aimed to describe the overall and surgery-specific incidence of POAF after noncardiac surgery and to investigate the association between preoperative $\mathrm{N}$-terminal pro-brain-type natriuretic peptide (NT-proBNP) and POAF. We hypothesized that measurement of NT-proBNP would improve POAF risk prediction beyond conventional prognostic factors.

\section{Methods}

\section{Study population}

The international VISION study included 40004 patients from 28 centres in 14 countries (Canada, the United States, Peru, Brazil, Colombia, South Africa, India, Malaysia, China, Australia, the United Kingdom, Spain, Poland and France) who underwent various noncardiac surgeries from August 2007 to November 2013 (ClinicalTrials.gov NCT00512109). All participating centres were university hospitals (Appendix 1, Supplementary Table 2). Informed consent was obtained before (in most cases) or within 24 hours of surgery from patients or their designated decisionmakers. Seven centres used a deferred consent process for patients who were unable to provide consent and for whom no designated decision-maker was available.

To ensure the representativeness of the study sample population, research personnel were responsible for daily screening of patient lists in preoperative assessment clinics and daily surgical lists to identify patients undergoing elective or urgent or emergent surgery during weekdays and weekends, as well as during the day and the night. ${ }^{22-24}$ Eligible patients were those aged 45 years or older who had inpatient noncardiac surgery. Preoperative blood samples were collected for NT-proBNP measurement from a subset of patients within selected centres.

\section{Data sources}

The selection of centres involved in the NT-proBNP substudy was based on their willingness to participate and the availability of the infrastructure to perform the collection, centrifugation and shipment of samples to our facility or to test locally. Five centres measured NT-proBNP locally: 1 centre performed NT-proBNP measurements in real time, whereas the other 4 sites batched their samples and later ran the assays at the same time. The remaining 11 centres centrifuged the collected samples, and shipped them to the Clinical Research Laboratory and Biobank in Hamilton, Ontario, Canada, where the samples were frozen and then later thawed to perform NT-proBNP measurements at the same time. Each laboratory performed its own quality control as a part of its standard procedures. Health care providers and study personnel were blinded to NT-proBNP measurements.

We calculated the Birmingham 2009 schema $\left(\mathrm{CHA}_{2} \mathrm{DS}_{2}-\mathrm{VASc}\right)$ score $^{25}$ to stratify the risk of thromboembolic events in patients who developed POAF. This tool is recommended by the American College of Cardiology, American Heart Association and European Society of Cardiology to assess indications for anticoagulation in patients with AF. The score incorporates age (1 point for age
65-74 yr, 2 points for age $\geq 75 \mathrm{yr}$ ) and sex ( 1 point for female sex), as well as history of congestive heart failure (1 point), hypertension (1 point), diabetes mellitus ( 1 point), vascular diseases ( 1 point) and previous thromboembolic events ( 2 points). ${ }^{26,27} \mathrm{~A}$ follow-up telephone call was conducted with patients or their relatives or caregivers 30 days after surgery, and we obtained outcome documentation in cases of new-onset POAF.

\section{Outcomes}

Our primary outcome was clinically important POAF within 30 days after surgery. There was no electrocardiogram (ECG) screening protocol to detect POAF, other than local clinical practice. Recorded POAF events were adjudicated by 2 expert physicians according to the VISION definition and using all available source documents. ${ }^{24}$ Clinically important POAF was defined as AF (ECG-documented) that resulted in angina, congestive heart failure or symptomatic hypotension or that required treatment with a rate-controlling drug, an antiarrhythmic drug or electrical cardioversion. This definition has previously been shown to be associated with an increased length of hospital stay, stroke and death. ${ }^{4,24,28}$ Patients were prospectively observed throughout the hospital stay by the medical staff, who recorded any event of clinically important POAF. Study personnel phoned each patient or patient's relative 30 days after the procedure to gather data concerning study outcomes.

\section{Statistical analysis}

We used data from a large cohort study (VISION) where sample size calculations were based on the expected incidence of major cardiac complications after noncardiac surgery. ${ }^{22}$ We performed a complete-case analysis owing to a relatively small fraction of missing data reported for the overall VISION cohort. ${ }^{22,24}$ We determined the proportion of patients who presented with POAF up to 30 days after surgery and the associated 95\% confidence intervals (Cls). We described the association between preoperative NT-proBNP modelled as a continuous variable and POAF using univariable and multivariable logistic regression models derived using data from 9789 patients who participated in the VISION preoperative NT-proBNP substudy.

We tested the added prognostic value of NT-proBNP over and above (i.e., independent of, after adjustment for) other potential predictors of POAF that were identified in the existing literature. Variable selection was guided by the authors' knowledge of the subject matter and the number of events available for analysis. The full list of variables that we identified through a literature search and initially considered as potential predictors of POAF is listed in Appendix 2, available at www.cmaj.ca/lookup/doi/10.1503/cmaj.200840/ tab-related-content. We focused on features that are routinely measured before surgery, to build the baseline model. After choosing the final list of variables to include in the multivariable logistic regression model, we applied no further model selection procedures.

Our final model included age, sex, history of hypertension, history of coronary artery disease, history of congestive heart failure, history of chronic obstructive pulmonary disease and type of surgery (divided into 3 categories: low risk, major nonthoracic and major thoracic). We modelled age and NT-proBNP using restricted cubic splines, allowing for nonlinear relationships and avoiding 
the known problems resulting from categorization of continuous variables. ${ }^{29}$ We used Wald tests to assess departures from linearity of log-odds. Splines are best interpreted using a figure, but they also allow comparison of any 2 desired values of the predictor that can be easily interpreted as an odds ratio (OR) without the need to refit the model. ${ }^{30}$ We summarized the added prognostic value of NT-proBNP as the fraction of new information (proportion of explainable variation that is explained by the biomarker; ratio of variances of predicted values before and after adding the biomarker to the model). In addition, we visualized the information provided by NT-proBNP measurement by analyzing pre- and post-test probabilities (Appendix 1). All analyses were performed using $\mathrm{R}$ version 3.6.0 (R Project) using the rms, givitiR and ggplot2 packages. Reporting of the data conforms to the STROBE statement (Appendix 1, Supplementary Table 3).

\section{Ethics approval}

The study complied with the Declaration of Helsinki and its protocol was approved by the institutional ethics board at each site.

\section{Results}

From the full VISION cohort, we excluded 2189 (5.5\%) patients with a history of AF, or with AF present before surgery, and 151 $(0.4 \%)$ patients with missing information on AF, leaving 37664 patients for the final analysis (Figure 1).

Table 1 reports demographic, clinical and surgical characteristics for the included patients, as well as laboratory measurement values stratified by the occurrence of POAF. Appendix 1, Supplementary Table 4 provides the same information in the subgroup of patients who had NT-proBNP measured before surgery.

The incidence of clinically important POAF after noncardiac surgery was $1.0 \%$ (95\% Cl 0.9-1.1). It was the highest after major thoracic surgery $(3.2 \% ; 95 \% \mathrm{Cl} 2.3-4.4)$, followed by major nonthoracic surgery $(1.3 \% ; 95 \% \mathrm{Cl} 1.2-1.5)$ and low-risk surgery $(0.2 \%$; $95 \% \mathrm{Cl} 0.1-0.3)$. Most cases of POAF occurred during the initial hospital stay $(357,96.7 \%)$, with a median time to event of 2 days.

Treatment prompted by POAF occurred in 349 (94.6\%) patients, $67.7 \%$ of whom were treated with rate-controlling drugs, $55.7 \%$ with antiarrhythmic drugs and $6.5 \%$ with electrical cardioversion. A histogram showing distribution of $\mathrm{CHA}_{2} \mathrm{DS}_{2}-\mathrm{VASC}$ scores in this group is presented in Appendix 1 , Supplemental Figure 1. After subtracting points for sex from the total score, 336 of $369(91.1 \%)$ patients still had at least 1 point. Anticoagulation therapy was started in $21.8 \%$ of patients with POAF, and $2.7 \%$ were discharged home with such treatment.

We obtained preoperative NT-proBNP measurements for 9789 patients (26.0\%). A comparison of demographic and clinical characteristics of patients with and without preoperative NTproBNP measurements is presented in Appendix 1, Supplementary Table 5. We observed 109 POAF events in this subgroup. The median (interquartile range [IQR]) preoperative level of NT-proBNP was $258 \mathrm{ng} / \mathrm{L}$ (IQR 108-679) in patients who developed POAF and $86 \mathrm{ng} / \mathrm{L}$ (IQR 37-220) in those who did not develop POAF $(p<0.001)$. Histograms of preoperative NT-proBNP values stratified by the occurrence of POAF are provided in Figure 2. The unadjusted association

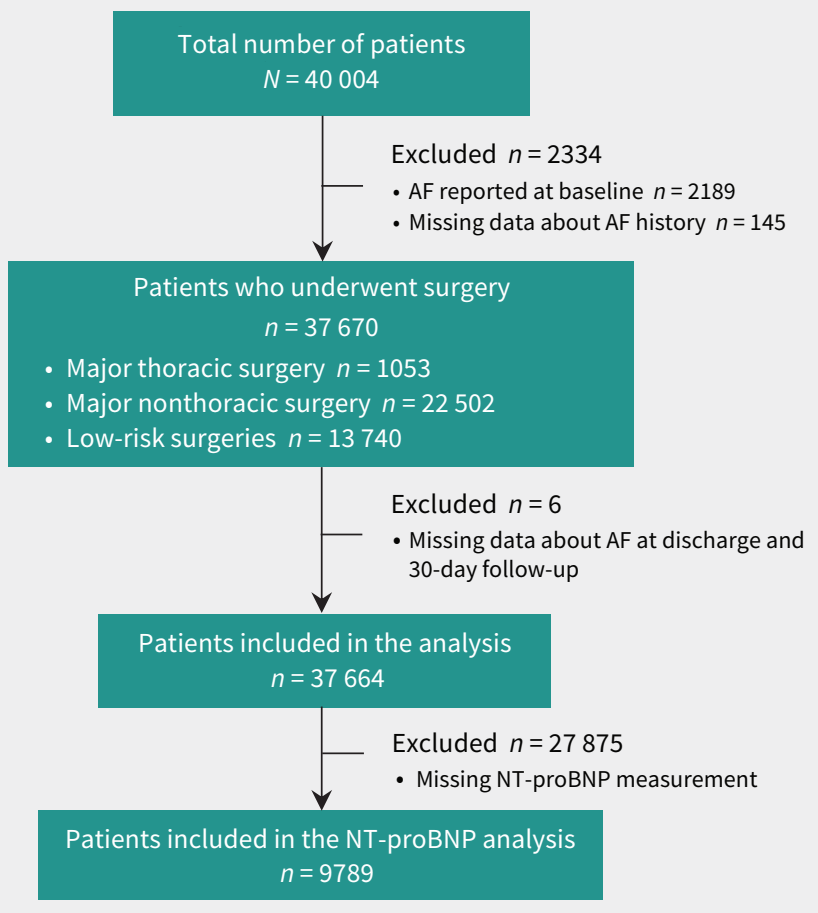

Figure 1: Flow chart for selection of study patients. Note: $\mathrm{AF}=$ atrial fibrillation, NT-proBNP = N-terminal pro-brain-type natriuretic peptide. 
Table 1: Patient demographic and clinical characteristics stratified by occurrence of new-onset, clinically important postoperative atrial fibrillation*

\begin{tabular}{|c|c|c|c|}
\hline Characteristic & $\begin{array}{l}\text { No. (\%) of patients without } \\
\text { POAF within } 30 \mathrm{~d} \text { after surgery† } \\
\qquad n=37295\end{array}$ & $\begin{array}{c}\text { No. (\%) of patients with POAF } \\
\text { within } 30 \mathrm{~d} \text { after surgery† } \\
n=369\end{array}$ & $\begin{array}{l}p \text { value for } \\
\text { difference }\end{array}$ \\
\hline Age, yr; mean \pm SD & $63.4 \pm 11.1$ & $73.1 \pm 10.2$ & $<0.001$ \\
\hline Female & $18724(50.2)$ & $169(45.8)$ & 0.09 \\
\hline Frailf & $2052(5.5)$ & $36(9.8)$ & 0.001 \\
\hline \multicolumn{4}{|l|}{ Smoking history } \\
\hline Current smoker & $20069(53.9)$ & $143(38.8)$ & $<0.001$ \\
\hline Former smoker & $11916(32.0)$ & $182(49.3)$ & \\
\hline \multicolumn{4}{|l|}{ Medical history } \\
\hline Coronary artery disease & 4250 (11.4) & $68(18.4)$ & $<0.001$ \\
\hline Recent high-risk CAD & $310(0.8)$ & $3(0.8)$ & $>0.99$ \\
\hline Congestive heart failure & $895(2.4)$ & $17(4.6)$ & 0.02 \\
\hline Aortic stenosis & $283(0.8)$ & $10(2.7)$ & $<0.001$ \\
\hline Peripheral vascular disease & $2759(7.4)$ & $44(11.9)$ & 0.002 \\
\hline History of CVE & $2134(5.7)$ & $38(10.3)$ & $<0.001$ \\
\hline History of DVT/PE & $1150(3.1)$ & $11(3.0)$ & $>0.99$ \\
\hline Hypertension & $18197(48.8)$ & $247(66.9)$ & $<0.001$ \\
\hline Diabetes & $7657(20.5)$ & $73(19.8)$ & 0.8 \\
\hline Chronic obstructive pulmonary disease & $2683(7.2)$ & $62(16.8)$ & $<0.001$ \\
\hline Obstructive sleep apnea & $1743(4.7)$ & $18(4.9)$ & 0.8 \\
\hline \multicolumn{4}{|l|}{ Preoperative medications $\leq 24 \mathrm{~h}$ before surgery } \\
\hline$\beta$-blockers & $\begin{array}{c}5386(14.5) \\
n=37260\end{array}$ & $\begin{array}{c}71(19.2) \\
n=369\end{array}$ & 0.01 \\
\hline Rate-controlling calcium channel blocker & $\begin{array}{c}694(1.9) \\
n=37264\end{array}$ & $\begin{array}{l}12(3.3) \\
n=369\end{array}$ & 0.08 \\
\hline \multicolumn{4}{|c|}{ Preoperative medications $>24 \mathrm{~h}$ to $7 \mathrm{~d}$ before surgery } \\
\hline$\beta$-blockers & $6032(16.2)$ & $84(22.8)$ & 0.001 \\
\hline Rate-controlling calcium-channel blocker & $819(2.2)$ & $15(4.1)$ & 0.03 \\
\hline Urgent/emergent surgery & $3792(10.2)$ & $62(16.8)$ & $<0.001$ \\
\hline \multicolumn{4}{|l|}{ Surgery } \\
\hline Thoracic surgery & $1053(2.8)$ & $35(9.5)$ & \\
\hline Major nonthoracic surgery & $22502(60.3)$ & $306(82.9)$ & $<0.001$ \\
\hline Other surgeries & $13740(36.8)$ & $28(7.6)$ & \\
\hline \multicolumn{4}{|l|}{ Type of anesthesia } \\
\hline General anesthesia & $22961(61.7)$ & $187(50.7)$ & \\
\hline Regional anesthesia & $10145(27.2)$ & $75(20.3)$ & $<0.001$ \\
\hline Combined general and regional anesthesia & $4137(11.1)$ & $107(29.0)$ & \\
\hline \multicolumn{4}{|l|}{ Preoperative hemoglobin, g/L } \\
\hline Mean \pm SD & $\begin{array}{c}130.4 \pm 19.4 \\
n=35978\end{array}$ & $\begin{array}{c}125.8 \pm 21.1 \\
n=364\end{array}$ & \\
\hline Median (IQR) & $\begin{array}{c}132(120-143) \\
n=35978\end{array}$ & $\begin{array}{c}128(113-140) \\
n=364\end{array}$ & $<0.001$ \\
\hline \multicolumn{4}{|l|}{ Preoperative eGFR, $\mathrm{mL} / \mathrm{min} / 1.73 \mathrm{~m}^{2}$} \\
\hline Mean \pm SD & $\begin{array}{c}80.5 \pm 24.2 \\
n=34696\end{array}$ & $\begin{array}{c}68.6 \pm 25.4 \\
n=361\end{array}$ & \\
\hline Median (IQR) & $\begin{array}{c}84.7(67.8-97.2) \\
n=34696\end{array}$ & $\begin{array}{c}73.3(52.2-88.3) \\
n=361\end{array}$ & $<0.001$ \\
\hline
\end{tabular}

between NT-proBNP levels and POAF is shown in Figure 3. We used an NT-proBNP level of $100 \mathrm{ng} / \mathrm{L}$ as a reference value to obtain ORs from logistic regression models. We found that preoperative NT-
proBNP levels of 200, 1500 and $3000 \mathrm{ng} / \mathrm{L}$ were associated with unadjusted ORs for POAF equal to 1.50 (95\% Cl 1.33-1.69), 2.75 (95\% Cl 1.81-4.20) and $3.32(95 \% \mathrm{Cl} 1.90-5.80)$, respectively. The 


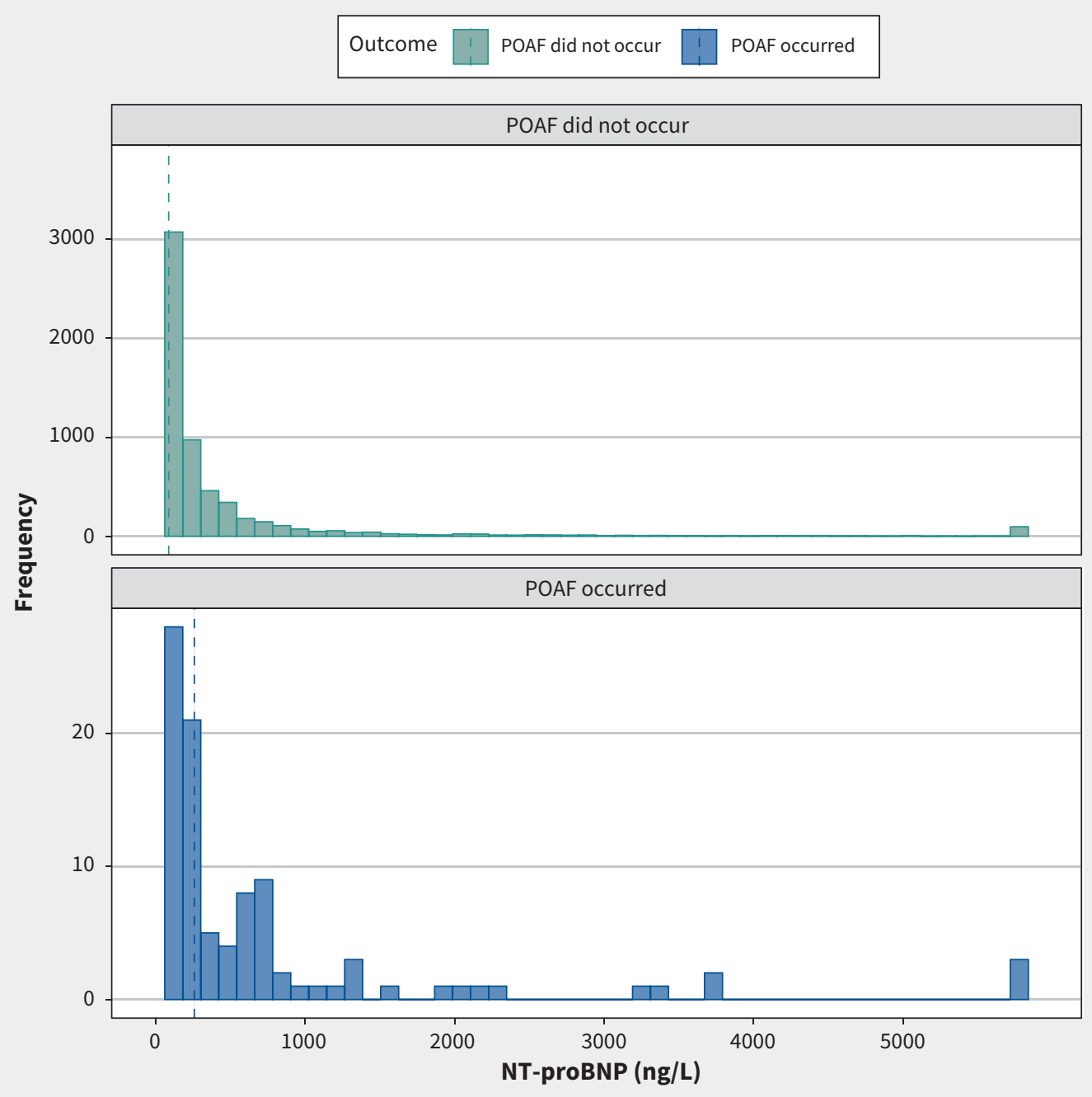

Figure 2: Histograms presenting distributions of values of N-terminal pro-brain-type natriuretic peptide (NT-proBNP) stratified by the occurrence of postoperative atrial fibrillation (POAF). Median values of NT-proBNP in both groups are marked with broken lines. Values of NT-proBNP winsorized at the 99th percentile (i.e., 98 most extreme values replaced with $5732 \mathrm{ng} / \mathrm{L}$ ) for increased readability.

multivariable adjusted associations are shown in Figure 3 and Table 2. We determined that preoperative NT-proBNP levels of 200, 1500 and $3000 \mathrm{ng} / \mathrm{L}$ were associated with adjusted ORs for POAF of 1.31 (95\% Cl 1.15-1.49), 2.07 (95\% Cl 1.27-3.36) and $2.39(95 \% \mathrm{Cl}$ 1.26-4.51), respectively. The fraction of new information from NTproBNP over and above other prognostic factors was equal to $16 \%$. Graphical representation of information provided by the biomarker can be seen in Appendix 1, along with model internal validation and goodness-of-fit measures.

\section{Interpretation}

We found that new, clinically important POAF complicates 1 in 100 major noncardiac surgeries, with a rate of 2 events per 1000 patients after low-risk surgery and 32 events per 1000 patients after major thoracic surgery. Importantly, these numbers represent the incidence of overt AF events that manifested with clinical symptoms or required treatment in the postoperative period. We also found that elevated levels of preoperative
NT-proBNP were associated with increased odds of POAF and provided additional information independent of other established prognostic factors. Postoperative atrial fibrillation prompted anticoagulation treatment in only $22 \%$ of patients, and only a small fraction of these patients continued the treatment after hospital discharge.

The reported incidence of POAF varies across different studies (Appendix 1, Supplementary Table 1). Our results are consistent with those of large registry-based studies. A retrospective cohort study of administrative data from a cohort of more than 1.6 million patients in California showed an incidence of POAF equal to $3.7 \%$ in patients undergoing thoracic procedures and $0.7 \%$ in patients after nonthoracic procedures, ${ }^{3}$ whereas a retrospective review involving 363092 patients who underwent major noncardiac surgery, $2.2 \%$ of whom underwent thoracic surgery, in 375 hospitals in the United States found that POAF complicated $1 \%$ of all procedures. ${ }^{2}$ In a 2018 Danish registry study that included more than 1.5 million patients undergoing both thoracic and nonthoracic noncardiac surgery, the incidence of POAF 

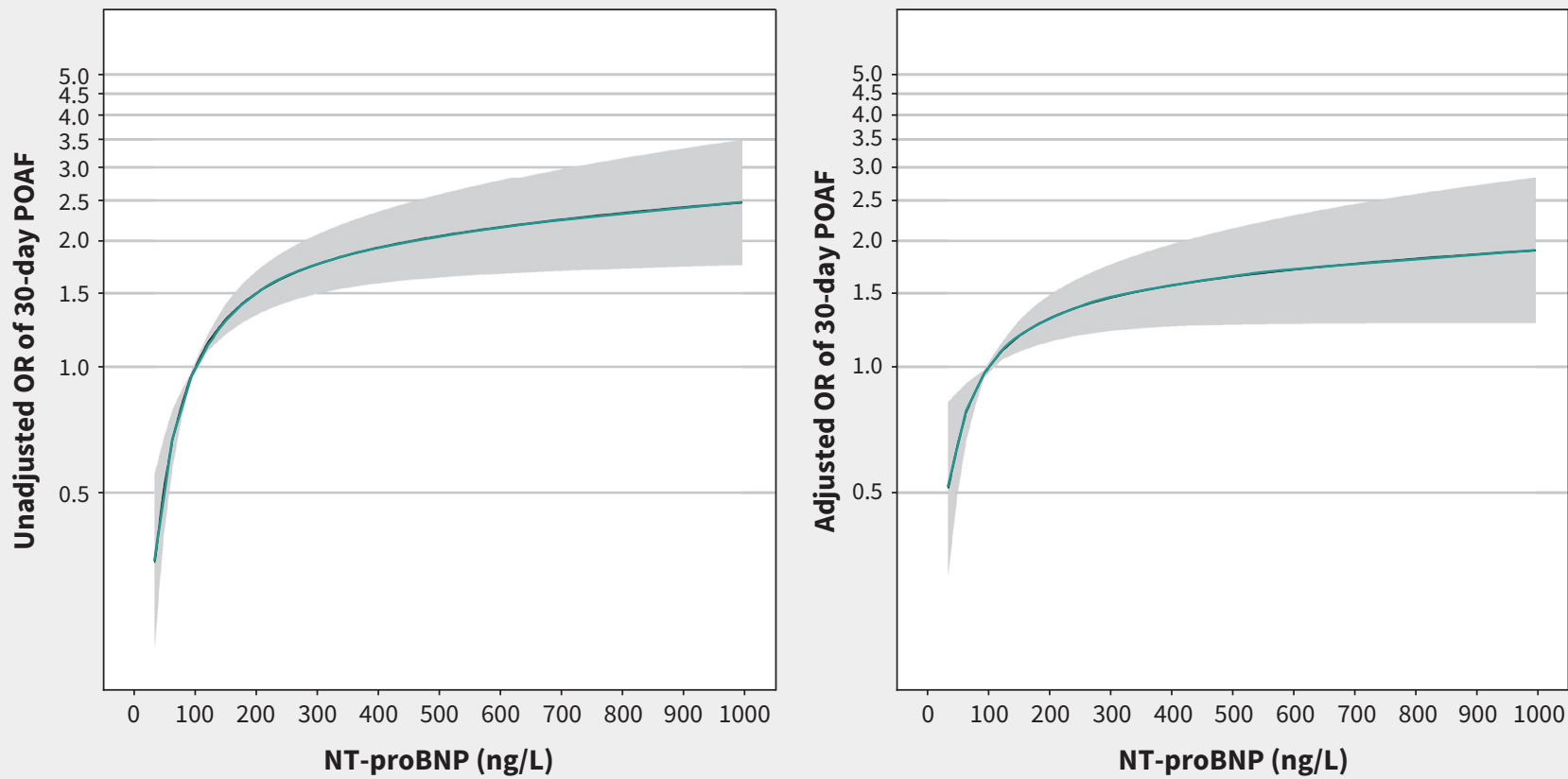

Figure 3: Unadjusted (left) and adjusted (right) relation between N-terminal pro-brain-type natriuretic peptide (NT-proBNP) measurement and postoperative atrial fibrillation (POAF). The test for nonlinearity of the estimated unadjusted relation between NT-proBNP and POAF had a $p$ value $=0.01$. The test for nonlinearity of the estimated relation had a $p$ value $=0.2$ in the multivariable analysis. The grey area represents $95 \%$ confidence intervals. Note: $O R=0 d d s$ ratio.

was $2.7 \%$ and $0.4 \%$, respectively. ${ }^{7}$ The similar incidence of POAF in administrative database studies and the VISION study may reflect that only postoperative AF with symptoms or requiring treatment is recorded in administrative databases (i.e., the VISION definition of POAF).

Preoperative NT-proBNP measurements were available in almost 10000 patients. Our study adds to the current understanding of the association between NT-proBNP and POAF by displaying the entire relation between preoperative levels of this biomarker and the occurrence of POAF without any arbitrary categorization. The prospective design and representative sample of our study provided an opportunity to assess the prognostic value of NT-proBNP measurement for the occurrence of clinically important POAF after noncardiac surgery. We found that NTproBNP provided important new information for POAF prediction independent of other prognostic factors.

The use of NT-proBNP is likely to increase. Elevated NT-proBNP before surgery is associated with increased risk of major perioperative cardiovascular outcomes after noncardiac surgery (e.g., vascular death, nonfatal myocardial infarction or myocardial injury), and the biomarker was shown to improve the predictive performance of the Revised Cardiac Risk Index..$^{31,32}$ The association between NT-proBNP and POAF after cardiac surgery has been researched extensively. ${ }^{19,33-36}$ Our results confirm the prognostic value of this biomarker in the setting of noncardiac surgery.

In our study, only $22 \%$ of the patients with POAF received anticoagulation therapy during the index hospital stay, which is consistent with other published studies. ${ }^{7,37}$ Less than $3 \%$ of patients with POAF were prescribed therapeutic anticoagulation therapy at the time of discharge from hospital, despite more than $90 \%$ of these patients having baseline $\mathrm{CHA}_{2} \mathrm{DS}_{2}$-VASc scores that would likely prompt the start of such therapy in a nonsurgical setting. ${ }^{27}$ There are no high-quality data on the proportion of patients with POAF who develop recurrent or chronic AF, which limits physicians' ability to make informed decisions on anticoagulation treatment for these patients. Data from the PeriOperative ISchemic Evaluation (POISE)-1 and POISE-2 trials showed an increased 1-year risk of stroke among patients who developed POAF, which suggests that such patients may benefit from anticoagulation therapy. ${ }^{28} \mathrm{~A}$ recent meta-analysis confirmed these findings. ${ }^{8}$ However, no randomized controlled trials to date have assessed the efficacy and safety of providing anticoagulation treatment for patients with POAF, and we believe that there is a clear need for such a trial. ${ }^{38}$

\section{Limitations}

We used the definition of clinically important POAF to capture new postoperative events that have immediate clinical consequences. It is likely that shorter episodes of mainly asymptomatic POAF are more frequent among noncardiac surgery patients and would have been detected more often with active screening. ${ }^{21}$ Similarly, extended monitoring after hospital discharge with remote devices could have shown a substantially higher rate of AF in the 30-day postoperative period. Continuous cardiac monitoring is, however, rarely used outside operating rooms and intensive care units, and the clinical consequences of asymptomatic POAF are not clear. Despite showing the added prognostic value of NT-proBNP, our analyses did not address the question about causal relations between various cardiovascular diseases leading to cardiac wall stress and, in turn, elevated levels of the biomarker and POAF. 
Table 2: Multivariable logistic regression model used to predict postoperative atrial fibrillation $(n=109)$ in a subgroup of 9789 patients who had NT-proBNP measured before surgery*

\section{Variable}

NT-proBNP, ng/L

100

200

1500

3000

Age, yr

50

65

80

$2.06(0.92-4.61)$

$4.37(1.91-9.98)$

Type of surgery

Low-risk

1 (Ref.)

Major nonthoracic

Major thoracic

$4.71(1.57-14.14)$

Demographic and clinical characteristics

Male sex

Hypertension

Diabetes mellitus

$1.21(0.76-1.92)$

Coronary artery disease

$1.12(0.69-1.81)$

Chronic obstructive

pulmonary disease
Note: $\mathrm{Cl}=$ confidence interval, $\mathrm{NT}$-proBNP $=\mathrm{N}$-terminal pro-brain-type natriuretic peptide, $\mathrm{OR}=$ odds ratio, Ref. $=$ reference category.

${ }^{*}$ We modelled NT-proBNP and age as continuous variables using restricted cubic splines. Splines are best interpreted using a figure, but they also allow the comparison of any 2 desired values of the predictor that can be easily interpreted as an OR without the need to refit the model. We used previously established thresholds for NT-proBNP and clinically meaningful increments for age. Odds ratios presented in the table can be interpreted as relative risks owing to the low baseline risk of the outcome.

\section{Conclusion}

We found that incidence of clinically important POAF after noncardiac surgery was $1 \%$. We also determined that preoperative NT-proBNP measurement was an independent prognostic factor for POAF. More research is needed to establish evidence-based prevention and treatment strategies for POAF in patients undergoing noncardiac surgery.

\section{References}

1. Hollenberg SM, Dellinger RP. Noncardiac surgery: postoperative arrhythmias. Crit Care Med 2000;28(Suppl 10):N145-50.

2. Bhave PD, Goldman LE, Vittinghoff E, et al. Incidence, predictors, and outcomes associated with postoperative atrial fibrillation after major noncardiac surgery. Am Heart J 2012;164:918-24.

3. Gialdini G, Nearing K, Bhave PD, et al. Perioperative atrial fibrillation and the long-term risk of ischemic stroke. JAMA 2014;312:616-22.

4. Alonso-Coello P, Cook D, Xu SC, et al. Predictors, prognosis, and management of new clinically important atrial fibrillation after noncardiac surgery: a prospective cohort study. Anesth Analg 2017;125:162-9.

5. Polanczyk CA, Goldman L, Marcantonio ER, et al. Supraventricular arrhythmia in patients having noncardiac surgery: clinical correlates and effect on length of stay. Ann Intern Med 1998;129:279-85.
6. Sohn GH, Shin DH, Byun KM, et al. The incidence and predictors of postoperative atrial fibrillation after noncardiothoracic surgery. Korean Circ $J$ 2009;39:100-4.

7. Butt JH, Olesen JB, Havers-Borgersen E, et al. Risk of thromboembolism associated with atrial fibrillation following noncardiac surgery. J Am Coll Cardiol 2018;72:2027-36.

8. Lin MH, Kamel H, Singer DE, et al. Perioperative/postoperative atrial fibrillation and risk of subsequent stroke and/or mortality. Stroke 2019;50:1364-71.

9. Roselli EE, Murthy SC, Rice TW, et al. Atrial fibrillation complicating lung cancer resection. J Thorac Cardiovasc Surg 2005;130:438-44.

10. Rao VP, Addae-Boateng E, Barua A, et al. Age and neo-adjuvant chemotherapy increase the risk of atrial fibrillation following oesophagectomy. Eur J Cardiothorac Surg 2012;42:438-43.

11. Brathwaite D, Weissman C. The new onset of atrial arrhythmias following major noncardiothoracic surgery is associated with increased mortality. Chest 1998;114:462-8.

12. Daniels LB, Maisel AS. Natriuretic peptides. J Am Coll Cardiol 2007;50:2357-68.

13. Wang TJ, Larson MG, Levy D, et al. Plasma natriuretic peptide levels and the risk of cardiovascular events and death. N Engl J Med 2004;350:655-63.

14. de Lemos JA, Ayers CR, Levine B, et al. Multimodality strategy for cardiovascular risk assessment: performance in 2 population-based cohorts. Circulation 2017;135:2119-32.

15. Patton KK, Ellinor PT, Heckbert SR, et al. N-terminal pro-B-type natriuretic peptide is a major predictor of the development of atrial fibrillation: the Cardiovascular Health Study. Circulation 2009;120:1768-74.

16. Sinner MF, Stepas KA, Moser CB, et al. B-type natriuretic peptide and C-reactive protein in the prediction of atrial fibrillation risk: the CHARGE-AF Consortium of community-based cohort studies. Europace 2014;16:1426-33.

17. Svennberg E, Lindahl B, Berglund L, et al. NT-proBNP is a powerful predictor for incident atrial fibrillation - validation of a multimarker approach. Int J Cardiol 2016;223:74-81.

18. Takase H, Dohi Y, Sonoda H, et al. Prediction of atrial fibrillation by B-type natriuretic peptide. J Atr Fibrillation 2013;5:674.

19. Cai GL, Chen J, Hu CB, et al. Value of plasma brain natriuretic peptide levels for predicting postoperative atrial fibrillation: a systemic review and meta-analysis. World J Surg 2014;38:51-9.

20. Simmers D, Potgieter D, Ryan L, et al. The use of preoperative B-type natriuretic peptide as a predictor of atrial fibrillation after thoracic surgery: systematic review and meta-analysis. J Cardiothorac Vasc Anesth 2015;29:389-95.

21. Higuchi S, Kabeya Y, Matsushita K, et al. Incidence and complications of perioperative atrial fibrillation after non-cardiac surgery for malignancy. PLoS One 2019;14:e0216239.

22. Writing Committee for the VISION Study Investigators; Devereaux PJ, Biccard $\mathrm{BM}$, et al. Association of postoperative high-sensitivity troponin levels with myocardial injury and 30-day mortality among patients undergoing noncardiac surgery. JAMA 2017;317:1642-51.

23. Botto F, Alonso-Coello P, Chan MT, et al. Myocardial injury after noncardiac surgery: a large, international, prospective cohort study establishing diagnostic criteria, characteristics, predictors, and 30-day outcomes. Anesthesiology 2014;120:564-78.

24. The Vascular Events In Noncardiac Surgery Patients Cohort Evaluation (VISION) Study Investigators. Association between postoperative troponin levels and 30-day mortality among patients undergoing noncardiac surgery. JAMA 2012;307:2295-304.

25. Lip GY, Nieuwlaat R, Pisters R, et al. Refining clinical risk stratification for predicting stroke and thromboembolism in atrial fibrillation using a novel risk factor-based approach: the Euro Heart Survey on atrial fibrillation. Chest 2010;137:263-72.

26. January CT, Wann LS, Calkins H, et al. 2019 AHA/ACC/HRS focused update of the 2014 AHA/ACC/HRS guideline for the management of patients with atrial fibrillation: a report of the American College of Cardiology/American Heart Association Task Force on Clinical Practice Guidelines and the Heart Rhythm Society in collaboration with the Society of Thoracic Surgeons. Circulation 2019;140:e125-51.

27. Kirchhof P, Benussi S, Kotecha D, et al. 2016 ESC guidelines for the management of atrial fibrillation developed in collaboration with EACTS. Eur Heart $J$ 2016;37:2893-962.

28. Conen D, Alonso-Coello P, Douketis J, et al. Risk of stroke and other adverse outcomes in patients with perioperative atrial fibrillation 1 year after non-cardiac surgery. Eur Heart J 2020;41:645-51.

29. Altman DG, Royston P. The cost of dichotomising continuous variables. BMJ 2006;332:1080.

30. Shepherd BE, Rebeiro PF, Caribbean C. South America network for HIVe. Brief report: assessing and interpreting the association between continuous covariates and outcomes in observational studies of HIV using splines. J Acquir Immune Defic Syndr 2017;74:e60-3. 
31. Rodseth RN, Biccard BM, Le Manach Y, et al. The prognostic value of preoperative and post-operative B-type natriuretic peptides in patients undergoing noncardiac surgery: B-type natriuretic peptide and $\mathrm{N}$-terminal fragment of pro-B-type natriuretic peptide: a systematic review and individual patient data meta-analysis. J Am Coll Cardiol 2014;63:170-80.

32. Duceppe E, Patel A, Chan MTV, et al. Preoperative N-terminal pro-B-type natriuretic peptide and cardiovascular events after noncardiac surgery: a cohort study. Ann Intern Med 2020;172:96-104.

33. Wazni OM, Martin DO, Marrouche NF, et al. Plasma B-type natriuretic peptide levels predict postoperative atrial fibrillation in patients undergoing cardiac surgery. Circulation 2004;110:124-7.

34. Cardinale D, Colombo A, Sandri MT, et al. Increased perioperative N-terminal pro-B-type natriuretic peptide levels predict atrial fibrillation after thoracic surgery for lung cancer. Circulation 2007;115:1339-44.
35. Amar D, Zhang H, Tan KS, et al. A brain natriuretic peptide-based prediction model for atrial fibrillation after thoracic surgery: development and internal validation. J Thorac Cardiovasc Surg 2019;157:2493-9 e1.

36. Amar D, Zhang $\mathrm{H}$, Shi $\mathrm{W}$, et al. Brain natriuretic peptide and risk of atrial fibrillation after thoracic surgery. J Thorac Cardiovasc Surg 2012;144: 1249-53.

37. Kirby A, Jayathissa S. New atrial fibrillation diagnosed perioperativelyanticoagulation practices in a secondary hospital. N Z Med J 2018;131: 72-8.

38. McIntyre WF, Healey JS, Devereaux PJ, et al. A call for randomized trials of oral anticoagulation for patients with post-operative atrial fibrillation. J Am Coll Cardiol 2019;73:1105.
Competing interests: P.J. Devereaux has received grants from Abbott Diagnostics, Boehringer Ingelheim, Roche Diagnostics and Siemens, as well as a grant and products from Philips Healthcare outside the submitted work. Emmanuelle Duceppe has received an investigator-initiated grant and a donation-inkind (assays) from Roche Diagnostics during the conduct of the study. She also received an investigator-initiated grant and a donation-inkind (assays) from Abbott Laboratories, and an investigator-initiated grant from Boehringer Ingelheim outside of the submitted work. Pablo Alonso-Coello's institution received funding from the Instituto de Salud Carlos III (Madrid, Spain) and Fundació La Marató de TV3 (Esplugues de Llobregat, Spain). No other competing interests were declared.

This article has been peer reviewed.

Affiliations: Department of Intensive Care and Perioperative Medicine (Szczeklik, Fronczek, Polok, Górka), Jagiellonian University Medical College, Kraków, Poland; Population Health Research Institute (LeManach, Conen, Duceppe, Pettit, Devereaux) and Department of Health Research Methods, Evidence, and Impact (LeManach, Conen, Heels-Ansdell), McMaster University, Hamilton, Ont.; Division of General Internal Medicine, Department of
Medicine (McAlister), University of Alberta, Edmonton, Alta.; Department of Surgery (Srinathan), University of Manitoba, Winnipeg, Man.; Iberoamerican Cochrane Center (AlonsoCoello), Biomedical Research Institute Sant Pau (IIB-Sant Pau-CIBERESP), Barcelona, Spain; Department of Anaesthesia and Perioperative Medicine (Biccard), Groote Schuur Hospital and University of Cape Town, South Africa; Division of Nephrology (Roshanov), Department of Medicine, London Health Sciences Centre, London, Ont.

Contributors: All of the authors contributed substantially to the conception or design of the work; or the acquisition, analysis or interpretation of data for the work; drafted the work or revised it critically for important intellectual content; gave final approval of the version to be published; and agreed to be accountable for all aspects of the work in ensuring that questions related to the accuracy or integrity of any part of the work are appropriately investigated and resolved.

${ }^{*}$ Dr. Yannick LeManach died on July 12, 2020, during preparation of this manuscript for publication.

Funding: This work was supported by more than 60 grants and funding sources (see
Appendix 3 for full list, available at www.cmaj. ca/lookup/doi/10.1503/cmaj.200840/tab-related -content). No funding entity had a role in data collection, statistical analysis, manuscript writing or the decision to publish.

Data sharing: The Vascular events In noncardiac Surgery patlents cOhort evaluatioN (VISION) Study investigators prioritize access to data analyses to researchers who have worked on the study for a substantial duration, have played substantial roles and have participated in raising the funds to conduct the study. The VISION investigators balance the length of the research study, and the intellectual and financial investments that made it possible, with the need to allow wider access to the data collected. Data will be disclosed only upon request and approval of the proposed use of the data by a Review Committee. Data are available to the journal for evaluation of reported analyses. Data requests from other non-VISION investigators will not be considered until 7 years after the close out of the study.

Accepted: Aug. 5, 2020

Correspondence to: Wojciech Szczeklik, wojciech.szczeklik@uj.edu.pl 